\title{
Vlasov simulations of parallel potential drops
}

\author{
H. Gunell ${ }^{1}$, J. De Keyser ${ }^{1}$, E. Gamby ${ }^{1}$, and I. Mann ${ }^{2,3}$ \\ ${ }^{1}$ Belgian Institute for Space Aeronomy, Avenue Circulaire 3, 1180 Brussels, Belgium \\ ${ }^{2}$ EISCAT Scientific Association, P.O. Box 812, 98128 Kiruna, Sweden \\ ${ }^{3}$ Department of Physics, Umeå University, 90187 Umeå, Sweden \\ Correspondence to: H. Gunell (herbert.gunell@physics.org)
}

Received: 29 April 2013 - Accepted: 18 June 2013 - Published: 18 July 2013

\begin{abstract}
An auroral flux tube is modelled from the magnetospheric equator to the ionosphere using Vlasov simulations. Starting from an initial state, the evolution of the plasma on the flux tube is followed in time. It is found that when applying a voltage between the ends of the flux tube, about two thirds of the potential drop is concentrated in a thin double layer at approximately one Earth radius altitude. The remaining part is situated in an extended region 1-2 Earth radii above the double layer. Waves on the ion timescale develop above the double layer, and they move toward higher altitude at approximately the ion acoustic speed. These waves are seen both in the electric field and as perturbations of the ion and electron distributions, indicative of an instability. Electrons of magnetospheric origin become trapped between the magnetic mirror and the double layer during its formation. At low altitude, waves on electron timescales appear and are seen to be non-uniformly distributed in space. The temporal evolution of the potential profile and the total voltage affect the double layer altitude, which decreases with an increasing field aligned potential drop. A current-voltage relationship is found by running several simulations with different voltages over the system, and it agrees with the Knight relation reasonably well.
\end{abstract}

Keywords. Magnetospheric physics (auroral phenomena; electric fields); Space plasma physics (numerical simulation studies)

\section{Introduction}

Electric fields parallel to the magnetic field are known to exist in the auroral zone, and they contribute to the acceleration of auroral electrons. Transverse electric fields at high altitude result in parallel electric fields as a consequence of the closure of the field aligned currents through the conducting ionosphere (Lyons, 1980). These magnetic field aligned electric fields can be supported by the magnetic mirror field, giving rise to potential drops that extend over great distances in space (Alfvén and Fälthammar, 1963).

Early theory included stationary kinetic models of the potential profile and of the relationship between the fieldaligned current and the total potential drop along the field line (Knight, 1973). If the electrostatic potential $V$ is monotonic, the current $I$ carried between the ionosphere and the magnetospheric source can be calculated once the distribution functions at, and the voltage between, the two regions are known. Fridman and Lemaire (1980) found that for parameters of interest in the upward current region the current is proportional to the potential drop. Similar current-voltage characteristics were later found for plasmas with kappa distributions (Pierrard, 1996; Pierrard et al., 2007).

One particular difficulty, which was pointed out by Persson (1966), is that unique potential profiles $V(z)$ only can be found under certain conditions. This can be seen considering the effective potential $U(z)=q V(z)+\mu B(z)$, which was introduced by Whipple (1977). Here $q$ is the particle charge, $\mu$ its magnetic moment and $B$ the magnetic flux density. If $U(z)$ has no maximum between the source of the population and the point under consideration, the distribution function can be uniquely obtained from the distribution function at the source point. If, however, such a maximum exists for particles with certain values of $\mu$, the distribution function cannot be determined from the distribution function at the source point and the local quantities $V(z)$ and $B(z)$ alone. Chiu and Schulz (1978) studied the electrostatic potential as a function of the spatial coordinate along the magnetic field and, assuming a stationary state, showed that the condition for $U(z)$ having no maximum is that $d V / d B>0$ and $d^{2} V / d B^{2} \leq 0$. 
While a maximum in $U(z)$ is an effective potential barrier, a minimum constitutes an effective potential well, where there may be a trapped population of particles that are unable to reach both the equatorial and the ionospheric source points.

Auroral flux tubes have been modelled with various approaches. Fluid models have been used to study Alfvén waves, and build-up of the parallel electric field (Rönnmark and Hamrin, 2000). Combined fluid/kinetic approaches have also been used, where particle simulations provide non-local kinetic closure of the fluid equations (Vedin and Rönnmark, 2006). Static solutions to Vlasov's equation have been found for auroral flux tubes (Ergun et al., 2000) covering several Earth radii, and Vlasov simulations of double layers have been performed in shorter simulation regions (Main et al., 2006). Vlasov simulations have also been used to study the relationship between ion heating and outflow and electric double layers in the downward current region of the aurora (Hwang et al., 2009). Watt et al. (2004) used a drift-kinetic simulation model to study the effect of shear Alfvén wave pulses on the electron distribution, and they found both parallel electric fields and significant electron heating. Driftkinetic simulations of auroral field lines above $1.5 R_{\mathrm{E}}$ altitude showed that trapping of warm plasma sheet electron populations by shear Alfvén waves can prevent wave damping, allowing acceleration of auroral electrons (Watt and Rankin, 2009). Recent results from kinetic modelling of magnetic reconnection have shown similar phenomena, including localised electrostatic potential drops and trapped particles, albeit on spatial scales much smaller than in auroral acceleration (Egedal et al., 2009).

Observations of parallel electric fields and double layers in magnetised plasmas have been made in laboratory experiments (e.g. Torvén and Andersson, 1979; Torvén, 1982; Schrittwieser et al., 1992). Experiments on parallel electric fields in a magnetic mirror configuration were performed in a double-ended Q machine by Sato et al. (1986) and a singleended Q machine by Sato et al. (1988). This experiment was modelled using particle in cell simulations (Ishiguro et al., 1995). Song et al. (1992a) derived a stability criterion which shows how the double layer position can be stabilised by the converging magnetic field. This criterion was tested in a Q-machine experiment (Song et al., 1992b). Starting in the 1970s, the formation of potential structures and double layers was modelled using particle simulations (DeGroot et al., 1977; Sato and Okuda, 1980, 1981; Sato et al., 1995).

Later, double layers were studied through laboratory experiments and numerical simulations, showing how waves near the local plasma frequency can be concentrated in narrow oscillating electric field spikes (Gunell et al., 1996a,b; Gunell and Löfgren, 1997; Löfgren and Gunell, 1998), which are associated with whistler emissions (Brenning et al., 2006). The electron beams and whistlers in the latter experiment are similar to those recently observed at Saturn's moon Enceladus (Gurnett et al., 2011). Observations have shown that electric double layers play an important role in auroral physics (Ergun et al., 2002; Andersson et al., 2002). Twospacecraft measurements have revealed further details of the potential structure in the acceleration region (Marklund et al., 2011). Observations in the downward current region have shown that electric double layers can be stabilised by the presence of a suprathermal component of the electron distribution (Andersson et al., 2008). This was confirmed by modelling using Vlasov simulations (Newman et al., 2008a,b).

In this paper we present results from electrostatic $(\nabla \times \boldsymbol{E}=$ $-\partial \boldsymbol{B} / \partial t=0)$ Vlasov simulations that are one-dimensional in space and two-dimensional in velocity space. In Sect. 2 the simulation model is described. In Sect. 3 the model is used to simulate an auroral flux tube from the ionosphere to the magnetospheric equator. Finally, the conclusions are discussed in Sect. 4.

\section{Simulation model}

\subsection{Geometry}

We model an auroral flux tube from a source at the magnetospheric equator to the ionosphere, as is illustrated in Fig. 1a. Figure $1 \mathrm{~b}$ shows a schematic picture of the cross section of an auroral arc. Such an arc can extend over distances on the order of $1000 \mathrm{~km}$ perpendicular to the plane of the figure. At the centre of the arc the electric and magnetic fields are parallel. Off centre, the perpendicular electric field component causes the plasma to drift along the arc with a drift velocity $\boldsymbol{v}_{\mathrm{d}}=\boldsymbol{E} \times \boldsymbol{B} / B^{2}$. In the following sections all equations are written for the centre of the arc, where there is no perpendicular electric field component and no drift. For positions off centre, the equations are still valid in a frame of reference that moves with the plasma drift velocity $\boldsymbol{v}_{\mathrm{d}}$. In that frame the perpendicular electric field component is zero (see also De Keyser and Echim, 2010; De Keyser et al., 2010). Since we model only the dimension along the magnetic field, the condition for our model to be valid at non-zero drifts is that the drift (and therefore also the electric field) is uniform along the arc, as a non-uniform drift in the dimension perpendicular to $\boldsymbol{B}$ would cause compressions and rarefactions that are not part of the model. The drift speed and the length of the arc determine the transit time for the drifting plasma, and that sets a limit to the timescales that can be modelled, or for a given timescale it sets the limit on what drift speeds can be accepted. Thus, from these geometrical considerations we conclude that the model is always valid for the centre of the arc where the perpendicular electric field component is zero, and where there is a perpendicular electric field component it is valid in the drifting frame of reference as long as the drift speed is not too large.

\subsection{Vlasov equation}

In our description of the problem, we write the distribution function as $f\left(z, v_{z}, \mu, t\right)$, where $z$ is the spatial coordinate 


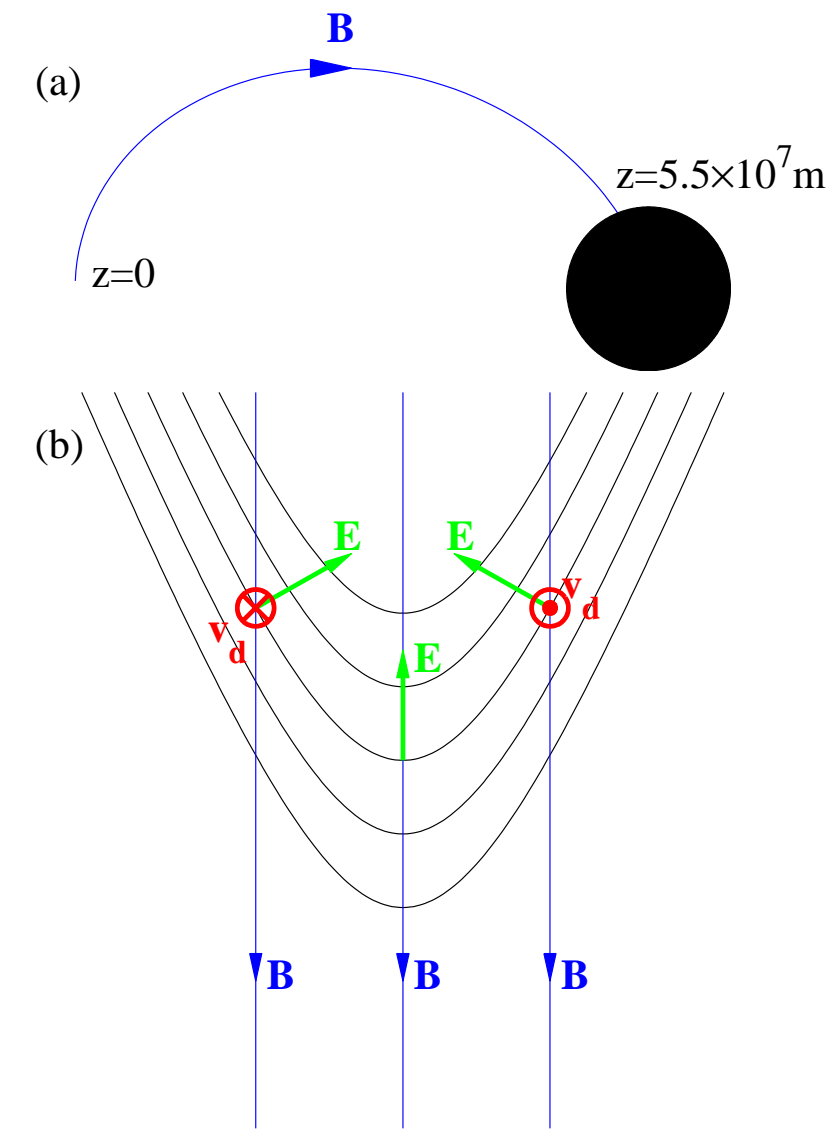

(c)

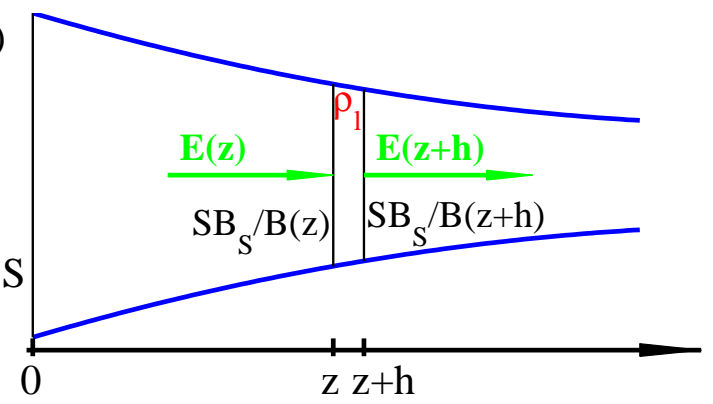

Fig. 1. Schematic of the geometry. (a) The plasma on a magnetic flux tube is modelled between the equatorial magnetosphere, where $z=0$, and the ionosphere at $z=5.5 \times 10^{7} \mathrm{~m}$. (b) Closeup of the cross section of an auroral arc. The black curves are equipotential lines. At the centre of the arc the electric and magnetic fields are parallel. Off centre, there is an $\boldsymbol{E} \times \boldsymbol{B}$ drift along the arc, into the plane of the paper on the left-hand side of the figure, and out of the plane on the right. (c) Illustration of the application of Gauss's law to a flux tube segment. The net charge in the segment is $\rho_{l} h$, and the flux tube cross section is $S B_{\mathrm{S}} / B(z)$, which reduces to $S$ at $z=0$.

parallel to the magnetic field, $v_{z}$ is the parallel velocity, and $\mu=\frac{m v_{\perp}^{2}}{2 B(z)}$

is the magnetic moment. Our model is, thus, one-dimensional in configuration space and two-dimensional in velocity space, where the perpendicular velocity is represented by the magnetic moment. With these restrictions, the only electric field component considered is parallel to the magnetic field. Each particle species satisfies a Vlasov equation:

$$
\frac{\partial f}{\partial t}+v_{\mathrm{z}} \frac{\partial f}{\partial z}+\frac{1}{m}\left(q E-\mu \frac{d B}{d z}+m a_{\mathrm{g}}\right) \frac{\partial f}{\partial v_{\mathrm{z}}}+\dot{\mu} \frac{\partial f}{\partial \mu}=0,
$$

where $a_{\mathrm{g}}$ is the component of the gravitational acceleration that is parallel to the magnetic field. If we assume that $\mu$ is a constant of motion,

$\dot{\mu}=0$.

This is a good approximation as long as the change in $\boldsymbol{B}$ is small over one gyration, and its validity in a specific case can be verified by computing the change in $\mu$ using a threedimensional test particle simulation that does not rely on the assumption in Eq. (2). Thus, throughout the paper the Vlasov equation we consider is

$\frac{\partial f}{\partial t}+v_{\mathrm{z}} \frac{\partial f}{\partial z}+\frac{1}{m}\left(q E-\mu \frac{d B}{d z}+m a_{\mathrm{g}}\right) \frac{\partial f}{\partial v_{\mathrm{z}}}=0$.

Equation (3) could be seen as an electrostatic version of the drift-kinetic equation that has been used to study Alfvén waves and their relationship to the aurora (Watt et al., 2004; Watt and Rankin, 2010).

To integrate Eq. (3) on a discrete spatial grid we use the flux conservative and positive scheme by Filbet et al. (2001). This is a variety of the splitting scheme originally proposed by Cheng and Knorr (1976). It is essentially a leap-frog scheme, where the phase space flux in the $z$ direction is computed at integral time steps and the flux in the $v_{\mathrm{z}}$ direction is computed at half time steps. Computing the flux in one of the phase space dimensions and updating the distribution function accordingly is usually referred to as an advection step. In this particular scheme, a third-order reconstruction of a primitive function of the distribution function is used in the computation of the flux. Limiters of the slope of the primitive function are used to restrict the distribution function to the interval $0 \leq f \leq f_{\infty}$, where $f_{\infty}$ is the global maximum of the distribution function. For more information see the original paper by Filbet et al. (2001) and also Schmitz and Grauer (2006).

We write the distribution function as a function of $z, v_{\mathrm{z}}$, and $\mu$, and only consider the $z$ component of the magnetic field. When $\mu$ is a constant of motion, we can compute the flux in velocity space in one single step in the $v_{z}$ direction, and there is no flux in the $\mu$ direction. The velocity step in $v_{z}$ is given by

$$
\Delta v_{\mathrm{z}}=\left(\frac{q E}{m}-\frac{\mu}{m} \frac{d B}{d z}+a_{\mathrm{g}}\right) \Delta t .
$$

The gravitational acceleration depends on the configuration that we seek to model. In the case of auroral field lines, the 
component along the magnetic field is used. The altitude dependence is given by Newton's gravitational law and the inclination of the magnetic field.

Since there is no flux in the $\mu$ direction, the simulated distribution function can be considered as a collection of independent parallel systems, only linked through the electric field. Filbet et al. (2001) used one global maximum value of the distribution function, denoted $f_{\infty}$, to compute the limiters that ensure positivity. In our case, separate maximum values, one for each $\mu$ value, are used, since the distribution for each value of $\mu$ is a separate system.

\subsection{Electric field calculation}

Applying Gauss's law to a flux tube segment with no perpendicular electric fields, as illustrated in Fig. 1c, we have

$E(z+h) S B_{\mathrm{S}} / B(z+h)-E(z) S B_{\mathrm{S}} / B(z)=\left(\rho_{l} / \epsilon\right) h$,

where $S$ is the flux tube cross section at the reference point where $B=B_{\mathrm{S}}$. The charge per unit length of the flux tube is given by the line charge

$\rho_{\mathrm{l}}=\sum_{\mathrm{s}} q_{\mathrm{s}} \int f_{\mathrm{s}}\left(v_{\mathrm{z}}, \mu\right) d \mu d v_{\mathrm{z}}$

where we form the sum of all species $s$ on the right-hand side. Rearranging Eq. (5), and taking the limit $h \rightarrow 0$, one arrives at an equation for the electric field:

$\frac{d}{d z}\left(\frac{B_{\mathrm{S}}}{B} E\right)=\frac{\rho_{1}}{S \epsilon}$.

In three dimensions, the flux tube has a finite extent perpendicular to the magnetic field and, the cross section being inversely proportional to $B$, the density of species $s$ is

$n_{\mathrm{s}}=\frac{B}{S B_{\mathrm{S}}} \int f_{\mathrm{s}}\left(v_{\mathrm{z}}, \mu\right) d \mu d v_{\mathrm{z}}$.

In the simulations presented in this paper the reference point was chosen to be at the magnetospheric source. With these definitions an isotropic Maxwellian at the magnetospheric end of the system with density $n_{\mathrm{s}}$ and temperature $T$ would be written

$f\left(z, v_{\mathrm{Z}}, \mu\right)=\frac{n_{\mathrm{S}} S B_{\mathrm{S}}}{\left(k_{B} T\right)^{3 / 2}} \sqrt{\frac{m}{2 \pi}} e^{-\left(m v_{\mathrm{Z}}^{2} / 2+\mu B_{\mathrm{S}}\right) /\left(k_{B} T\right)}$.

Since $f$ is proportional to $S$, so is $\rho_{1}$ in Eq. (6), and with $S$ appearing in the denominator on the right-hand side of Eq. (7) that quantity is cancelled there, as it is in Eq. (8). Thus, $S$ need not appear in the computer code, but it is kept in Eqs. (7) and (8) in the interest of dimensional correctness.

Equation (7) is integrated to obtain the electric field

$E(z)=\frac{1}{\epsilon} \frac{B}{B_{\mathrm{S}}} \int_{0}^{z} \rho_{l} d z^{\prime}+\frac{B}{B_{\mathrm{S}}} E_{\mathrm{S}}$, where $E_{\mathrm{S}}$ is a constant that is determined by the boundary conditions, and which is equal to the electric field at $z=0$. The electrostatic potential $V$ is obtained by integrating Eq. (10), and with $V=0$ at $z=0$ we have

$V(z)=-\frac{1}{\epsilon} \int_{0}^{z} \frac{B}{B_{\mathrm{S}}}\left(\int_{0}^{z^{\prime}} \rho_{l} d z^{\prime \prime}\right) d z^{\prime}-E_{\mathrm{S}} \int_{0}^{z} \frac{B}{B_{\mathrm{S}}} d z^{\prime}$.

Applying the boundary condition that $V=V_{\mathrm{i}}$ at the ionospheric end of the system, where $z=z_{\mathrm{i}}$, the constant of integration $E_{\mathrm{S}}$ becomes

$E_{\mathrm{S}}=-\frac{V_{\mathrm{i}}+\frac{1}{\epsilon} \int_{0}^{z_{\mathrm{i}}} \frac{B}{B_{\mathrm{S}}}\left(\int_{0}^{z} \rho_{l} d z^{\prime}\right) d z}{\int_{0}^{z_{\mathrm{i}}} \frac{B}{B_{\mathrm{S}}} d z}$.

If instead the electric field at $z=0$ is specified as a boundary condition, $E_{\mathrm{S}}$ will simply be that field, and if the field is specified at $z=z_{\mathrm{i}}$ we have

$E_{\mathrm{S}}=-\frac{1}{\epsilon} \int_{0}^{z_{\mathrm{i}}} \rho_{\mathrm{l}} d z+\frac{B_{\mathrm{S}}}{B_{\mathrm{i}}} E_{\mathrm{i}}$

The validity of using a one-dimensional electric field calculation rests on the assumption that the perpendicular electric field is negligible inside the modelled flux tube, which ensures that the potential is constant over the cross section. If there is a perpendicular electric field the model is still valid in the frame of reference moving with the plasma drift velocity $\boldsymbol{v}_{\mathrm{d}}=\boldsymbol{E} \times \boldsymbol{B} / B^{2}$, as discussed in Sect. 2.1. Satellite crossings of the auroral cavity show that the perpendicular electric field falls in thin layers at the edges of the cavity with small or zero field inside (Hull et al., 2003; Ergun et al., 2004). The perpendicular fields at the flux tube edges are stronger than the parallel field inside. However, being perpendicular to the magnetic field, they cannot move charged particles in or out of the flux tube, nor can they contribute to acceleration parallel to the magnetic field. Thus, although a three-dimensional model would be required to provide a complete picture of the flux tube, acceleration processes at the centre of it are adequately modelled in one dimension.

\subsection{Initial state}

A major problem is that the initial state of the system generally is unknown. A fast procedure for obtaining a suitable initial state is outlined here. By introducing an artificial relative dielectric constant $\epsilon_{\mathrm{r}}$ such that $\epsilon=\epsilon_{0} \epsilon_{\mathrm{r}}$ in Eq. (7), we can run a simulation on a coarser spatial grid and with a longer time step, because $\lambda_{\mathrm{D}} \sim \sqrt{\epsilon_{\mathrm{r}}}$ and $\omega_{\mathrm{p}} \sim 1 / \sqrt{\epsilon_{\mathrm{r}}}$ (Rönnmark and Hamrin, 2000). With

$\epsilon_{\mathrm{r}}=\max \left(1,\left(a \omega_{\mathrm{p}} \Delta t\right)^{2}\right)$

the plasma frequency will be reduced so that there are $2 \pi a$ time steps per plasma period. In Eq. (14) $\omega_{\mathrm{p}}$ is taken to be 
the maximum plasma frequency in the system, and the resulting $\epsilon_{\mathrm{r}}$ is applied uniformly everywhere. A consequence of introducing an $\epsilon_{\mathrm{r}}>1$ is that sharp gradients become less sharp. For waves, the decreased plasma frequency and the increased Debye length cause waves to appear at lower frequencies and longer wavelengths. This has a damping effect on waves since it increases the growth lengths and growth times. The effective Debye length for one species in our system is

$\lambda_{\mathrm{s}}=\sqrt{\frac{\epsilon_{0} \epsilon_{\mathrm{r}} k_{\mathrm{B}} T_{\mathrm{z}, \mathrm{s}}}{|q| n_{\mathrm{s}}}}$.

If electrons of different origin are treated as different species, the effective electron Debye length is

$\lambda_{\mathrm{e}}=\sqrt{\sum_{\alpha} \lambda_{\alpha}^{-2}}$,

where $\alpha$ denotes all species representing electrons.

In Sect. 3 below, a series of simulations are run, starting from a high value of $\epsilon_{\mathrm{r}}$ and letting it decrease toward more realistic values for each run. In this way the simulation region is allowed to fill up quickly when the $\epsilon_{\mathrm{r}}$ value is large and to converge toward a realistic solution as $\epsilon_{\mathrm{r}}$ is decreased. Such a solution constitutes a reasonable, although not unique, initial state for a time-accurate simulation of the system. Computational efficiency benefits from a decreased number of grid cells when the grid is made coarser by the introduction of $\epsilon_{\mathrm{r}}$.

\subsection{A non-uniform grid}

The number of grid cells can be reduced by the introduction of a non-uniform grid, thus having a high spatial resolution only where this is needed. Our grid is non-uniform with respect to the $z$ coordinate. This is accomplished by transforming the $z$ axis to the $\xi$ axis by the transformation

$z=g(\xi)$.

A transformed equation can then be solved on the uniform $\xi$ grid. The parallel velocity becomes

$v_{z}=\frac{d z}{d t}=\frac{d z}{d \xi} \frac{d \xi}{d t}=g^{\prime} v_{\xi}$,

and we shall require

$\frac{d z}{d \xi}=g^{\prime}>0$

Now, the distribution is a function of $\xi$ :

$f\left(z, v_{z}, \mu, t\right)=f\left(g(\xi), g^{\prime} v_{\xi}, \mu, t\right)$.

We transform the second term of Eq. (3) and obtain

$\frac{\partial f}{\partial t}+\frac{v_{\mathrm{z}}}{g^{\prime}} \frac{\partial f}{\partial \xi}+\frac{1}{m}\left(q E-\mu \frac{d B}{d z}+m a_{\mathrm{g}}\right) \frac{\partial f}{\partial v_{\mathrm{z}}}=0$.
It is not necessary to transform the other terms of Eq. (3). Since we know both $\xi$ and $z$, we can perform the advection step in space using the transformed term and the advection in velocity using the original $v_{\mathrm{z}}$ coordinate. Similarly, there is no need to transform Eq. (7). It can be integrated numerically, taking the non-uniform nature of the grid into account, i.e. using different values of $\Delta z$ for different values of $z$.

We use rational functions to represent $g^{\prime}$, which we normalise so that Eq. (21) is solved on a uniform grid ranging from $\xi=0$ to $\xi=1$. If $g^{\prime}$ is a function of the form

$g^{\prime}(\xi)=\frac{1}{P(\xi)}$,

where $P(\xi)$ is a polynomial, we can write

$g^{\prime}(\xi)=\sum_{j} \frac{a_{j}}{\xi-b_{j}}$,

where $b_{j}$ are the poles of $g^{\prime}(\xi)$ and $a_{j}$ are the residues at those poles. The transformation function $g(\xi)$ is found by integrating $g^{\prime}(\xi)$, so that

$g(\xi)=\sum_{j} a_{j} \ln \left(\xi-b_{j}\right)$.

We then normalise so that $\hat{g}(0)=z_{\min }$ and $\hat{g}(1)=z_{\max }$ and we have

$$
\begin{aligned}
\hat{g}(\xi)= & z_{\min }+\left(\sum_{j} a_{j} \ln \left(\xi-b_{j}\right)-\sum_{j} a_{j} \ln \left(-b_{j}\right)\right) \\
& \times \frac{z_{\max }-z_{\min }}{\sum_{j} a_{j} \ln \left(1-b_{j}\right)-\sum_{j} a_{j} \ln \left(-b_{j}\right)} .
\end{aligned}
$$

Since a sum of functions of the form given by Eq. (23) is itself a function of that form, we can construct $g^{\prime}(\xi)$ as a sum:

$g_{k}^{\prime}(\xi)=\sum_{k} \frac{1}{P_{k}(\xi)}$.

Using functions of this form has the advantage that we can use techniques from filter theory, where rational functions are used to describe the filter transfer function. This is particularly useful when modelling auroral acceleration, where the need for spatial resolution changes quickly between low altitudes dominated by ionospheric plasma and higher altitudes where the hot magnetospheric plasma dominates. Simple pole expansions that were developed to model plasma distribution functions are also of the form given by Eq. (26) and can be described by their poles and residues (Löfgren and Gunell, 1997).

In our implementation, we write the poles and residues to a file, which the simulation program reads at the start of each run, and then the grid is created according to Eq. (25). An example is shown in Fig. 2. The top panel shows the transform $z=g(\xi)$, and the bottom panel shows the step size $\Delta z$ (solid 

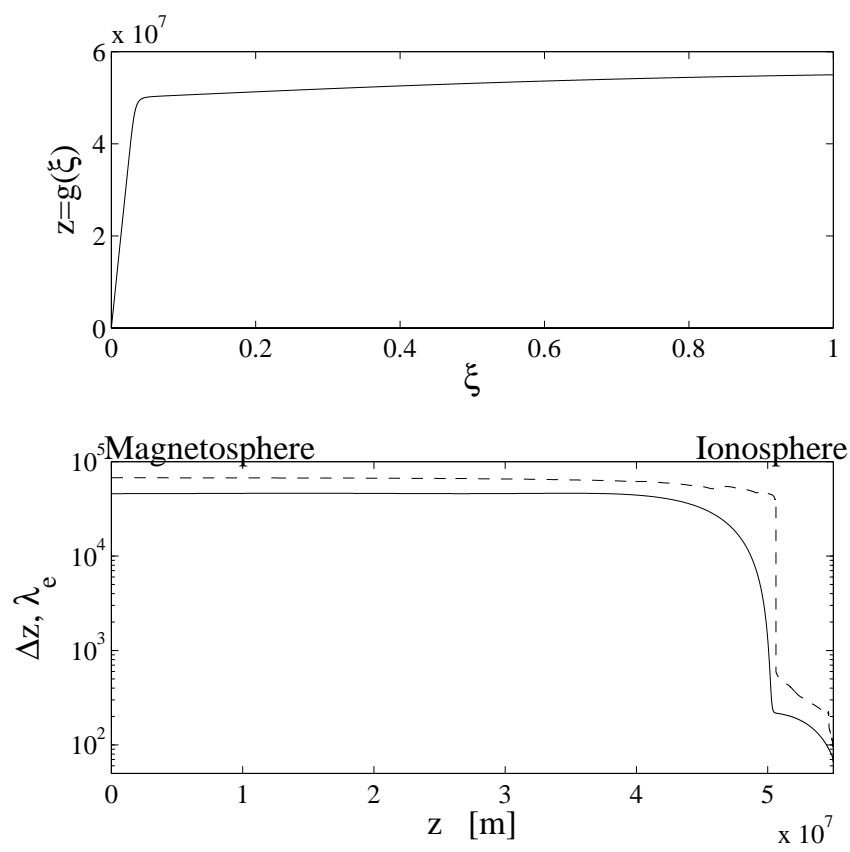

Fig. 2. An example of a transform: $z=g(\xi)$ (top) and the resulting step size $\Delta z$ (solid line) and the effective electron Debye length $\lambda_{\mathrm{e}}$ (dashed line), from a simulation, as functions of $z$ (bottom).

line), illustrating how the effective electron Debye length $\lambda_{\mathrm{e}}$ (dashed line) can be resolved. The transformation in this example is of the form

$g^{\prime}(\xi)=\frac{C_{1}}{P_{1}(\xi)}+\frac{C_{2}}{P_{2}(\xi)}+\frac{C_{3}}{P_{3}(\xi)}$,

where

$P_{1}(\xi)=1+\frac{1}{2}\left(\frac{\xi}{\xi_{1}}\right)^{2}+\frac{1}{2^{2} \cdot 2 !}\left(\frac{\xi}{\xi_{1}}\right)^{4}+\frac{1}{2^{3} \cdot 3 !}\left(\frac{\xi}{\xi_{1}}\right)^{6}$

is a truncated Taylor expansion of $\exp \left(\xi^{2} / \xi_{1}^{2}\right)$, and $\xi_{1}=0.6$ determines the slope of $\Delta z$ for $z \gtrsim 5 \times 10^{7} \mathrm{~m}$ as is seen in the lower panel of Fig. 2. The polynomial

$P_{2}(\xi)=1+0.1^{2} T_{4}^{2}\left(\frac{\xi}{\xi_{2}}\right)$

where $T_{4}\left(\xi / \xi_{2}\right)$ is a fourth-order Chebyshev polynomial, is responsible for the cutoff at $\xi=\xi_{2}=0.025$, corresponding to $z \approx 5 \times 10^{7} \mathrm{~m}$. This term is obtained from filter theory, where $1 / \sqrt{P_{2}(\xi)}$ is the transfer function of a fourth-order Chebyshev low-pass filter with $0.5 \%$ pass band ripple.

$$
P_{3}(\xi)=(\xi-1-10 i)(\xi-1+10 i)=\xi^{2}-2 \xi+101
$$

is a slowly varying function in $0 \leq \xi \leq 1$, which is added to the other two terms to adjust the minimum value of $\Delta z$. In this example, the values of the coefficients that appear in Eq. (27) are $C_{1} \approx 6.6 \times 10^{6} \mathrm{~m}, C_{2} \approx 1.5 \times 10^{9} \mathrm{~m}$, and
Table 1. Poles $\left(b_{j}\right)$ and residues $\left(a_{j}\right)$ of the transformation in Fig. 2. Only poles in the upper half-plane are included in this table.

\begin{tabular}{ccc}
\hline Group & $a_{j}$ & $b_{j}$ \\
\hline 1 & $0.0111+0.0062 i$ & $-0.6673+0.9750 i$ \\
1 & $-0.0111+0.0062 i$ & $0.6673+0.9750 i$ \\
1 & $0.0-0.0252 i$ & $0.0+1.0720 i$ \\
2 & $0.0275-0.0179 i$ & $-0.0299+0.0079 i$ \\
2 & $0.01114-0.0433 i$ & $-0.0124+0.0190 i$ \\
2 & $-0.0275-0.0179 i$ & $0.0299+0.0079 i$ \\
2 & $-0.0114-0.0433 i$ & $0.0124+0.0190 i$ \\
3 & $0.0-0.0239 i$ & $1.0+10 i$ \\
\hline
\end{tabular}

$C_{3} \approx 6.2 \times 10^{7} \mathrm{~m}$. Table 1 shows the poles and residues of the transformation illustrated in Fig. 2. Only the poles in the upper half-plane have been tabled. For each pole $b_{j}$ in the upper half-plane there is a complex conjugate pole $b_{j}^{*}$ in the lower half-plane, and its residue $a_{j}^{*}$ is the complex conjugate of $a_{j}$. The group number in the first column indicates to which one of $P_{1}, P_{2}$, and $P_{3}$ the pole belongs.

The grid can be made non-uniform also in the $\mu$ direction. As $\mu$ is an adiabatic invariant, this does not affect the solution of the Vlasov equation, since no advection step in $\mu$ is performed. The grid spacing in the $\mu$ direction, like in the $v_{\mathrm{z}}$ direction, can be different for the different species, and we have implemented a grid with grid cell boundaries at

$\mu_{j}=\mu_{\min }+\left(\mu_{\max }-\mu_{\min }\right)\left(\frac{j}{N_{\mu}}\right)^{\alpha}, \quad j=0,1,2, \ldots N_{\mu}$,

where $N_{\mu}$ is the number of grid cells in $\mu$ for the species in question and $\alpha$ is a constant that can be set for each species, and $\alpha=1$ yields a uniform grid. For the auroral simulations reported in this article we have used $\alpha=4$ and $\mu_{\min }=0$.

\subsection{Relativistic electrons}

When modelling large potential drops - that is to say, in the range of tens of kilovolts - electrons may reach relativistic speeds. For relativistic particles $\mu$ is no longer conserved. Instead the conserved quantity is (Alfvén and Fälthammar, 1963)

$\mu^{\prime}=\frac{p_{\perp}^{2}}{2 m_{0} B}=\frac{\gamma^{2} m_{0} v_{\perp}^{2}}{2 B}=\gamma \mu$,

where $m_{0}$ is the particle mass at rest, and

$\gamma=\frac{1}{\sqrt{1-\frac{v^{2}}{c^{2}}}}=\frac{1}{\sqrt{1-\frac{v_{z}^{2}+v_{\perp}^{2}}{c^{2}}}}$,

where $c$ is the speed of light in a vacuum. In order to implement a relativistic treatment in the code, we let $\mu^{\prime}$ replace 
$\mu$, so that the distribution function is $f\left(z, v_{\mathrm{Z}}, \mu^{\prime}, t\right)$ for those species that are treated relativistically. We are then able to compute $\gamma$ and re-write the $v_{\mathrm{z}}$ advection subroutine for these species, replacing $\Delta v_{\mathrm{z}}$ in Eq. (4) by

$\Delta v_{\mathrm{z}}=\left(\frac{q E}{\gamma m_{0}}-\frac{\mu^{\prime}}{\gamma^{2} m_{0}} \frac{d B}{d z}+a_{\mathrm{g}}\right) \Delta t$.

Solving the system given by Eqs. (32) and (33) yields an expression for $\gamma$ in our coordinates:

$\gamma=\sqrt{\frac{1+\frac{2 B \mu^{\prime}}{c^{2} m_{0}}}{1-\frac{v_{Z}^{2}}{c^{2}}}}$.

\subsection{Implementation}

The scheme described above has been implemented in Fortran code, and the computations have been parallelised using the Message Passing Interface (MPI). The $\xi$ axis is divided among the parallel processes in segments with as nearly equal a number of grid cells as possible. Each process exchanges messages with its two neighbours at each time step while solving the Vlasov equation (the two processes at the system ends only have one neighbour). The advection step in the $\xi$ dimension requires that the distribution function at the two $\xi$ points closest to the process boundary is sent to the neighbour process. Equation (7) is solved by a simple integration, where each process sends one message to process 0 and gets one back. Computing the flux and updating the distribution function are the most computer-intensive tasks.

Source code for the simulation program has been deposited with this article as supplementary material.

\section{Results}

We have used the simulation program to model the plasma on an auroral field line. The results are presented in this section.

Simulating an auroral flux tube, we use an approximation for the $L=7$ shell magnetic field (Vedin and Rönnmark, 2006)

$$
\begin{aligned}
B_{\mathrm{Z}}(z)= & B_{\mathrm{M}} \exp \left[( \frac { z } { L _ { \mathrm { z } } } ) ^ { 2 } \left(\ln \left(\frac{B_{\mathrm{I}}}{B_{\mathrm{M}}}\right)-0.6\right.\right. \\
& \left.\left.-1.8\left(\frac{z}{L_{\mathrm{z}}}\right)^{2}+2.4\left(\frac{z}{L_{\mathrm{z}}}\right)^{6}\right)\right],
\end{aligned}
$$

where $B_{\mathrm{I}}=56 \mu \mathrm{T}$ and $B_{\mathrm{M}}=0.0864 \mu \mathrm{T}$ are the magnetic flux densities at the ionospheric and the magnetospheric ends of the system respectively, and $L_{z}=5.5 \times 10^{7} \mathrm{~m}$ is the length of the system. The $z$ axis is defined so that $z=0$ at the magnetospheric end of the system, and $z=L_{\mathrm{z}}$ at the ionosphere. The magnetic field given by Eq. (36) is shown in Fig. 3e. The gravitational field is modelled by Newton's gravitational law,
Table 2. Parameters used in the simulations.

\begin{tabular}{lcc}
\hline & Magnetosphere & Ionosphere \\
\hline$z$ & 0 & $5.5 \times 10^{7} \mathrm{~m}$ \\
$B$ & $0.086 \mu \mathrm{T}$ & $56 \mu \mathrm{T}$ \\
$k_{\mathrm{B}} T_{\mathrm{e}}$ & $500 \mathrm{eV}$ & $1 \mathrm{eV}$ \\
$k_{\mathrm{B}} T_{\mathrm{H}}^{+}$ & $2500 \mathrm{eV}$ & $1 \mathrm{eV}$ \\
$n$ & $3 \times 10^{5} \mathrm{~m}^{-3}$ & $1 \times 10^{9} \mathrm{~m}^{-3}$ \\
\hline
\end{tabular}

and the component parallel to the magnetic field is found using a dipole model of the magnetic field line. Though this is done all along the $z$ axis, it is only at low altitude that the gravitational force is important.

We start with an empty simulation region at $t=0$. Maxwellian distributions are prescribed at the boundaries, and the system is allowed to fill up from the ends. This can be done quickly using a large $\epsilon_{\mathrm{r}}$ value. A series of simulation runs with successively lower $\epsilon_{\mathrm{r}}$ is performed, in order to produce a realistic initial state, starting from which a timeaccurate simulation of the system can be run.

A series of simulation runs was performed, starting with $\epsilon_{\mathrm{r}}=4.98 \times 10^{8}$, and with the boundary conditions that $V=0$ and $E=0$ at the magnetospheric end of the system. The ionospheric end was allowed to float. The simulation was run in this way for $10 \mathrm{~s}$, which corresponds to the time it takes a $2.5 \mathrm{keV}$ proton to travel $7 \times 10^{6} \mathrm{~m}$. Thus, it is shorter than the ion transit time by a factor of eight, but it allows the system to start filling up with plasma. Then the boundary conditions were changed by specifying the voltage over the system, and bringing it down linearly to $3 \mathrm{kV}$ during $20 \mathrm{~s}$. The simulation was run at $3 \mathrm{kV}$ until a steady state was reached after approximately five minutes. After that, three more runs were performed successively with decreasing $\epsilon_{\mathrm{r}}=1.27 \times 10^{7}, 7.96 \times 10^{5}$, and $4.98 \times 10^{4}$, each run starting from the plasma state at the end of the previous run. The magnetospheric electrons are treated relativistically, since they may be accelerated to relativistic speeds by transient electric fields when the simulation region is filled with plasma from the ends. The results from the last run are presented here. Plasma parameters for the magnetospheric and ionospheric boundaries are shown in Table 2.

Figure 3 shows (a) the plasma potential and (b) the densities of the different species, for the case where the total acceleration voltage was $3 \mathrm{kV}$. Figure $3 \mathrm{c}$ shows the plasma potential for each of the four different values of $\epsilon_{\mathrm{r}}$ mentioned above. The gradient steepens as $\epsilon_{\mathrm{r}}$ is decreased. The black curve in Fig. 3a shows the mean value of the potential during the interval $4.9 \mathrm{~s} \leq t \leq 5.0 \mathrm{~s}$. Here $t=0$ at the start of the run at $\epsilon_{\mathrm{r}}=4.98 \times 10^{4}$. Approximately two thirds of the potential drop is concentrated in a thin double layer just below one Earth radius altitude. The remaining kilovolt falls in a region that extends $1-2$ Earth radii above the double layer. Close to the double layer, at $z \approx 4.5 \times 10^{7} \mathrm{~m}$ and $z \approx 4.9 \times 10^{7} \mathrm{~m}$, 


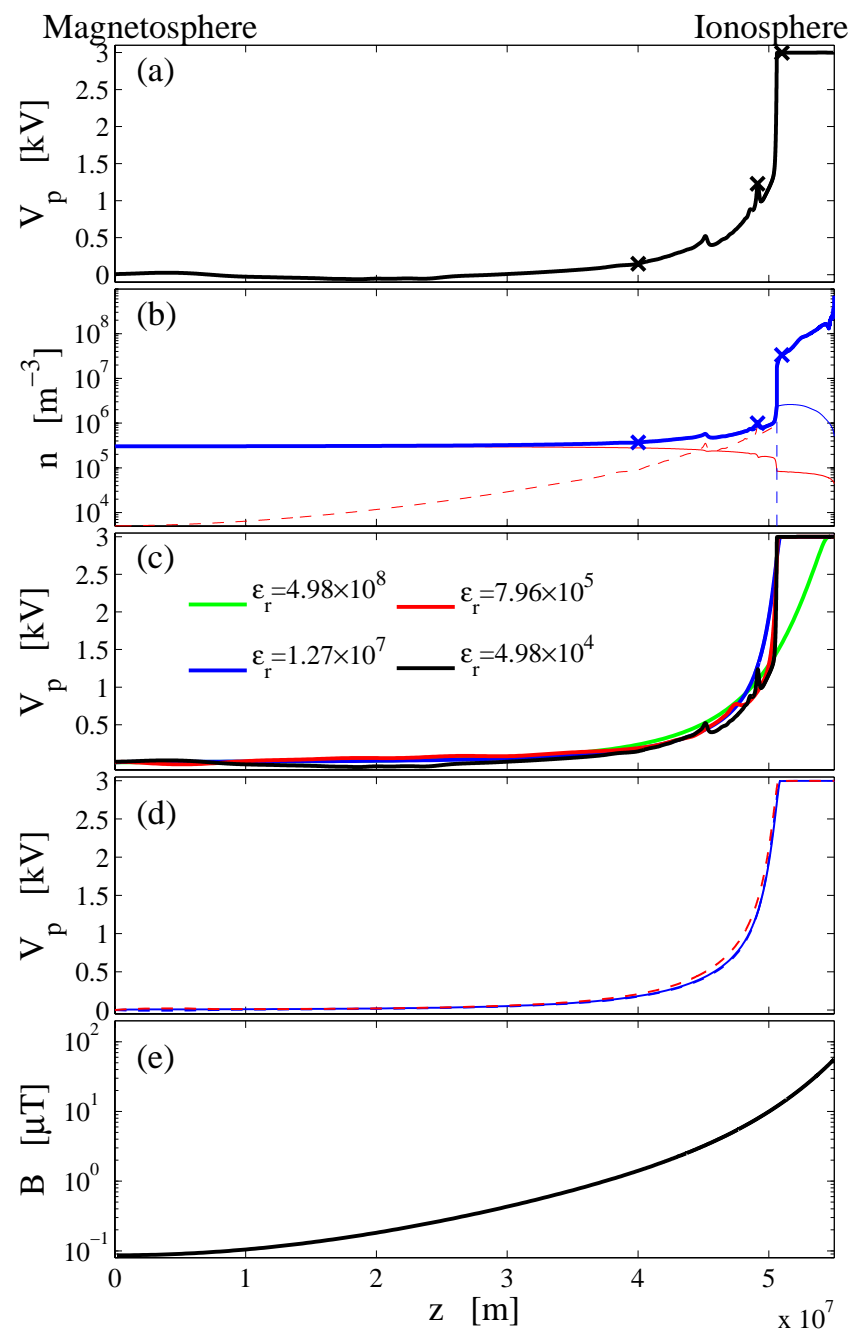

Fig. 3. (a) Plasma potential as a function of $z$. (b) The density in the same simulation. The thick blue curve shows the plasma density. The thin solid curves show protons (red) and electrons (blue) from the magnetospheric end of the system. The dashed curves show protons (red) and electrons (blue) originating from the ionosphere. The $\mathrm{Xs}$ indicate the positions for which distribution functions are shown in Fig. 6. (c) Potential profiles for different values of $\epsilon_{\mathrm{r}}$. (d) Test of the influence of the initialisation process. The dashed blue curve shows the potential after the system was filled at $\epsilon_{\mathrm{r}}=1.27 \times 10^{7}$. The dashed red curve shows the potential after the system was first filled at zero voltage, and then the voltage was ramped up to $3 \mathrm{kV}$, also at $\epsilon_{\mathrm{r}}=1.27 \times 10^{7}$. The solid blue curve is the corresponding potential profile copied from panel (c). The solid and dashed blue curves overlap almost completely. (e) Magnetic flux density $B$, as given by Eq. (36).

perturbations of the potential and density are seen. These move upward at a speed slightly above the ion acoustic speed of $250 \mathrm{~km} \mathrm{~s}^{-1}$, as is illustrated in Fig. 4, which shows a $z-t$ diagram of the electric field. The stationary double layer electric field is seen as a horizontal dark blue band across the fig-

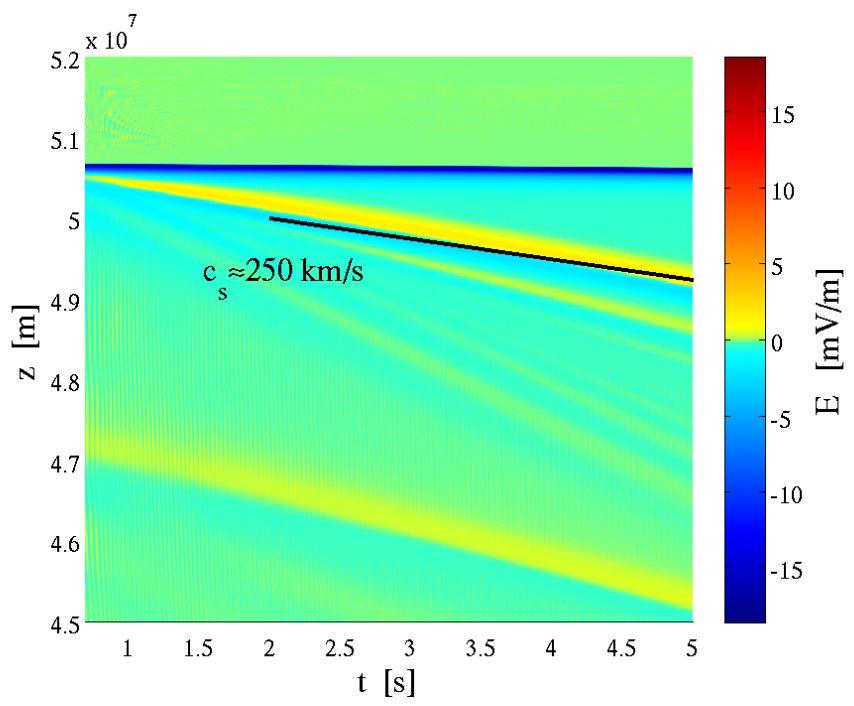

Fig. 4. The electric field in a $z-t$ diagram. The ion acoustic speed is indicated by the slope of the black line.

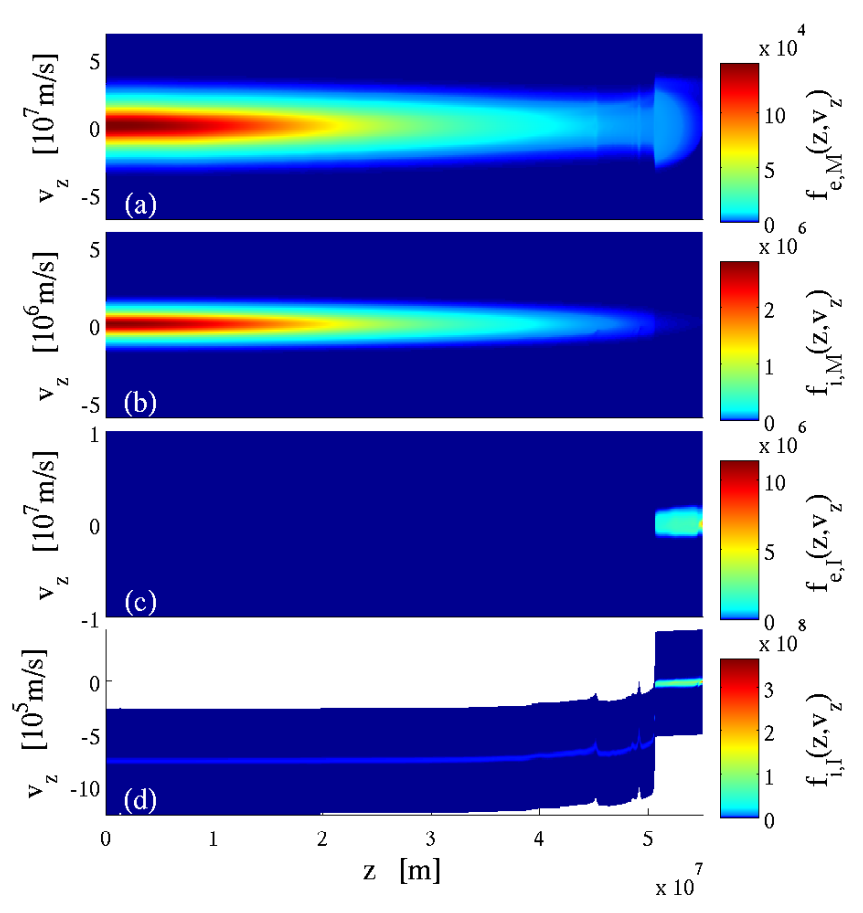

Fig. 5. Phase space densities $f\left(z, v_{\mathrm{Z}}\right)$ at $t=5.0 \mathrm{~s}$ for (a) magnetospheric electrons; (b) magnetospheric protons; (c) ionospheric electrons; and (d) ionospheric protons. The colour scales have been normalised so that integrals over all $v_{\mathrm{Z}}$ yield $n_{\mathrm{S}} / B$. In panel (d), the simulated velocity range is allowed to shift so that, for each value of $z$, it is centred on the mean velocity. Thus, large unpopulated phase space regions (white in this figure) do not need to be modelled.

ure. The ion acoustic waves are also seen as perturbations of the potential and density in Fig. 3.

In Fig. 3b, the thick blue curve shows the plasma density. The thin solid curves show protons (red) and electrons (blue) 

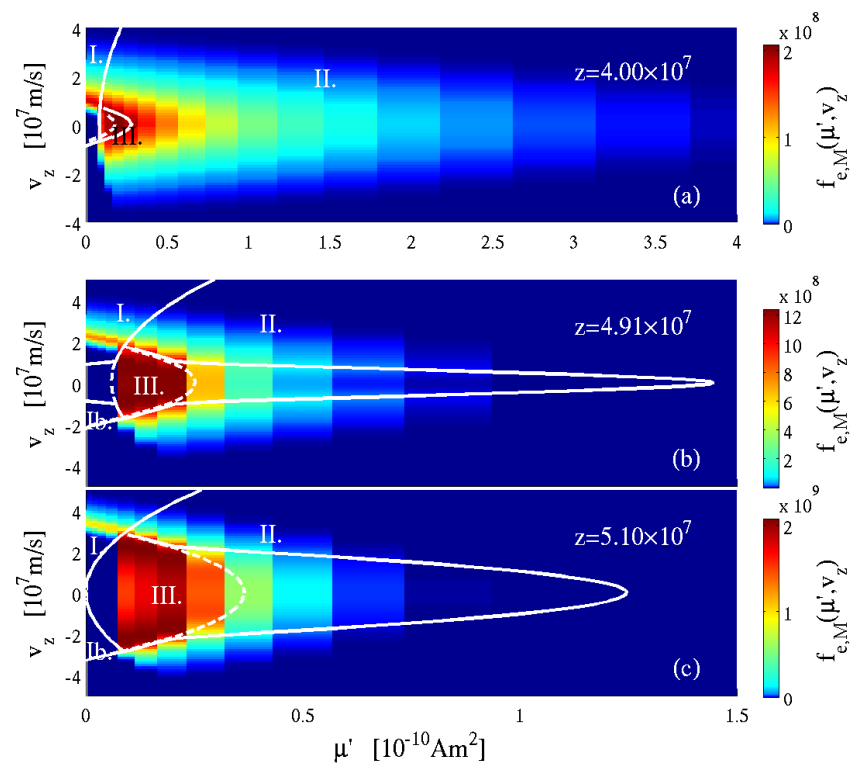

Fig. 6. Phase space densities $f\left(\mu^{\prime}, v_{\mathrm{Z}}\right)$ at $t=5.0 \mathrm{~s}$ for magnetospheric electrons at (a) $z=4 \times 10^{7} \mathrm{~m}$; (b) $z=4.91 \times 10^{7} \mathrm{~m}$; and (c) $z=5.1 \times 10^{7} \mathrm{~m}$. These positions are marked in the potential and density diagrams in Fig. 3. The colour scales have been normalised so that integrals over all of $\mu^{\prime}-v_{\mathrm{Z}}$ space yield $n_{\mathrm{S}}$. The regions indicated by white lines are I: precipitating electrons; Ib: up-going electrons that will reflect and then precipitate; II: electrons which can reach the equator; and III: trapped electrons. The dashed white lines show the boundaries between the regions computed according to the theory by Chiu and Schulz (1978), and the solid lines using Eqs. (37) and (38).

from the magnetospheric end of the system. The dashed curves show protons (red) and electrons (blue) originating from the ionosphere. A steep density gradient develops at the position of the double layer. This corresponds to the lower boundary of the auroral cavity. A less steep density gradient extends throughout the high-potential side of the double layer. In laboratory experiments, double layers are some tens of Debye lengths wide (Torvén and Andersson, 1979). For auroral double layers the difference between the Debye lengths on the two sides of the double layer is much larger than in laboratory plasmas, due to the larger difference in temperature. If we define the double layer width to be the distance over which the potential changes from $10 \%$ to $90 \%$ of the double layer voltage and take the double layer to be where the potential goes from $1000 \mathrm{~V}$ to $3000 \mathrm{~V}$, this width is 19 times $\lambda_{\mathrm{e}}$ on the high-altitude side. The Debye length on the high-altitude side is the relevant reference length, because on the other side, the much colder ionospheric plasma has only an insignificant influence on the double layer width. Thus the width is in agreement with laboratory experiments.

At the ionospheric boundary there is a sheath of about $1 \mathrm{~V}$, which is too small to be seen in Fig. 3a. It separates the cold dense ionospheric plasma at the boundary from the hotter and

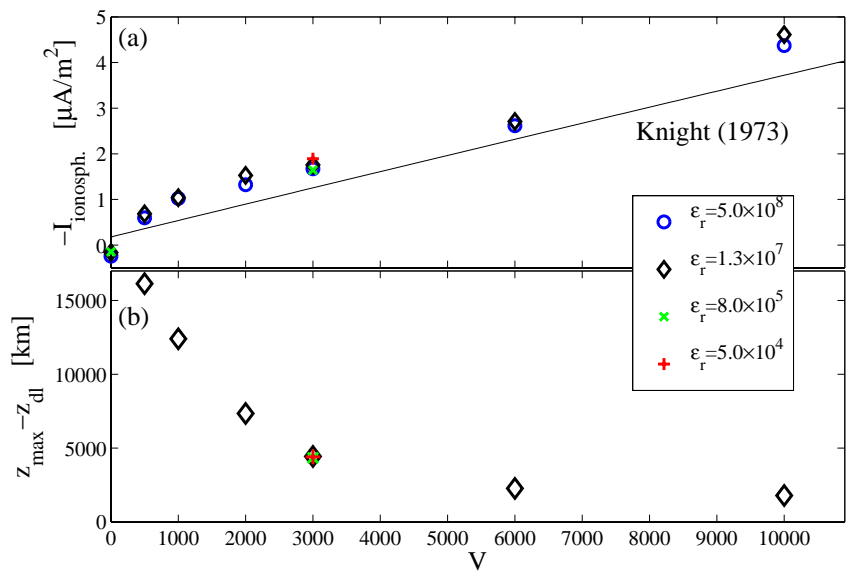

Fig. 7. (a) Current density scaled to the ionosphere as a function of the voltage across the system. The different symbols correspond to runs with different values of $\epsilon_{\mathrm{r}}$. At the two highest $\epsilon_{\mathrm{r}}$ values, $4.98 \times$ $10^{8}$ and $1.27 \times 10^{7}$, simulations have been run for all voltages. At $\epsilon_{\mathrm{r}}=1.27 \times 10^{7}$, simulations have been run for 0 and $3 \mathrm{kV}$, and at the lowest value, $\epsilon_{\mathrm{r}}=4.98 \times 10^{4}$, the simulation has been run only for a total voltage of $3 \mathrm{kV}$. The black line shows Knight's currentvoltage relationship. (b) Double layer position as a function of the total acceleration voltage.

thinner plasma above. Although the sheath voltage is similar to the ionospheric temperature, the velocity distributions in the interior are influenced less by the sheath than by wave heating above the ionosphere. The temperature of the ionospheric electrons increases from $1 \mathrm{eV}$ at the boundary to $3 \mathrm{eV}$ in the plasma between the double layer and the ionosphere.

To see how the initialisation process and the successive decreasing of $\epsilon_{\mathrm{r}}$ affect the results we have performed two tests. The dashed blue curve in Fig. 3d shows the potential after the system was filled from the ends at $\epsilon_{\mathrm{r}}=1.27 \times 10^{7}$. This should be compared to the solid blue curve, which was obtained as described above, filling the system first at $\epsilon_{\mathrm{r}}=$ $4.98 \times 10^{8}$ before decreasing $\epsilon_{\mathrm{r}}$ to $1.27 \times 10^{7}$. The dashed and the solid blue curves overlap to the extent that they cannot be distinguished in the figure. The dashed red curve in Fig. 3d shows the potential after the system was first filled at $0 \mathrm{~V}$, after which the voltage was ramped up to $3 \mathrm{kV}$ and the simulation was allowed to run until a steady state was reached. This test was run with $\epsilon_{\mathrm{r}}=1.27 \times 10^{7}$, and should be compared to the solid blue curve for that $\epsilon_{\mathrm{r}}$ value. The potential profiles are very similar for the different initialisation methods.

Figure 5 shows the distribution function $f\left(z, v_{\mathrm{z}}\right)$ at $t=$ $5.0 \mathrm{~s}$ for the different species: (a) magnetospheric electrons; (b) magnetospheric protons; (c) ionospheric electrons; and (d) ionospheric protons. Electrons that originate from the ionosphere (Fig. 5c) are confined to the low-altitude, highpotential, side of the double layer. On the right-hand side of Fig. $5 \mathrm{a}\left(z \gtrsim 5.1 \times 10^{7} \mathrm{~m}\right)$ one can see some electrons, coming from the magnetospheric end of the system, which have 
been trapped between the double layer and the magnetic mirror. The ionospheric protons (Fig. 5d) are first accelerated in the double layer, and then by the extended electric field at high altitude on the low-potential side. An ion instability can be seen developing at $z \approx 5 \times 10^{7} \mathrm{~m}$.

Figure 6 shows distribution functions $f\left(\mu^{\prime}, v_{\mathrm{Z}}\right)$ at $t=5.0 \mathrm{~s}$ for magnetospheric electrons at (a) $z=4 \times 10^{7} \mathrm{~m}$; (b) $z=$ $4.91 \times 10^{7} \mathrm{~m}$; and (c) $z=5.1 \times 10^{7} \mathrm{~m}$. These positions are marked in the potential and density diagrams in Fig. 3. White lines mark the boundaries between different phase space regions, assuming a stationary potential. Electrons in region I can reach the ionosphere, and region $\mathrm{Ib}$ contains up-going electrons that will be reflected and then join region I. Electrons in region II can reach the equator, either directly, or after being reflected by the magnetic mirror field. Region III contains electrons that are trapped. Applying the method proposed by Vedin and Rönnmark (2004) to our case, we find that a particle at $z=z_{1}$, where $V=V_{1}$ and $B=B_{1}$, can reach the ionosphere if

$\mu^{\prime} \leq \min _{z_{1}<z \leq L_{z}} \frac{1}{B(z)-B_{1}}\left(q\left(V_{1}-V(z)\right)+\frac{m v_{z 1}^{2}}{2}\right)$,

and for it to reach the equator it must have

$\mu^{\prime} \geq \max _{0 \leq z<z_{1}} \frac{1}{B(z)-B_{1}}\left(q\left(V_{1}-V(z)\right)+\frac{m v_{z 1}^{2}}{2}\right)$.

These boundaries are shown as solid white lines in Fig. 6. For comparison the boundaries derived under the condition that $d V / d B>0$ and $d^{2} V / d B^{2} \leq 0$ (Chiu and Schulz, 1978) are shown as dashed lines. These only take the $B$ and $V$ values at the boundaries and at the present location into account and not the places in between. Trapped populations of magnetospheric electrons, i.e. particles in region III, can be seen in Fig. 6.

Figure 7 a shows the simulated current-voltage relationship. The different symbols represent different values of $\epsilon_{\mathrm{r}}$, as indicated in the figure. These symbols overlap, showing the insensitivity of the current-voltage characteristic to the $\epsilon_{\mathrm{r}}$ value. At the two highest $\epsilon_{\mathrm{r}}$ values, $4.98 \times 10^{8}$ and $1.27 \times 10^{7}$, simulations have been run for $0,0.5,1,2,3,6$, and $10 \mathrm{kV}$. At $\epsilon_{\mathrm{r}}=1.27 \times 10^{7}$, simulations have been run for 0 and $3 \mathrm{kV}$, and at the lowest $\epsilon_{\mathrm{r}}$ value, $4.98 \times 10^{4}$, the simulation has been run for the $3 \mathrm{kV}$ case only. The solid line shows Knight's current-voltage relation (Knight, 1973). The currents obtained in these simulations match Knight's relation reasonably well.

Figure $7 \mathrm{~b}$ shows the double layer position as a function of the total acceleration voltage. It is shown as the height above the ionospheric end of the system $z_{\max }-z_{\mathrm{DL}}$, and it is taken to be the position of the most negative electric field. A welldefined double layer is found for the second-highest level of $\epsilon_{\mathrm{r}}$ values, i.e. $\epsilon_{\mathrm{r}}=1.27 \times 10^{7}$, and its position remains the same when $\epsilon_{\mathrm{r}}$ is decreased further. This is also seen in Fig. 3c. Higher voltages yield lower double layer altitudes.

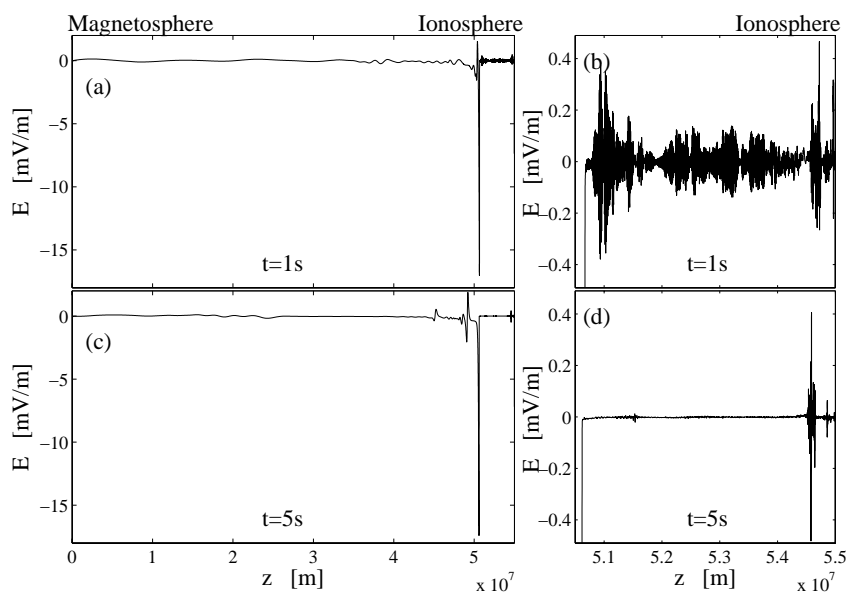

Fig. 8. (a) Electric field as a function of $z$ at $t=1 \mathrm{~s}$ for the case with $3 \mathrm{kV}$ total voltage. The narrow minimum near $z=5.1 \times 10^{7} \mathrm{~m}$ is the double layer electric field. (b) The electric field on the highpotential, low-altitude, side of the double layer at $t=1 \mathrm{~s}$. (c) Same as (a) but for $t=5 \mathrm{~s}$. (d) Same as (b) but for $t=5 \mathrm{~s}$.

Figure 8 shows the simulated electric field as a function of $z$ for $t=1 \mathrm{~s}$ and $t=5 \mathrm{~s}$. In panels (a) and (c) the full $z$ range is shown for these two instants in time. The large negative electric field near $z=5.1 \times 10^{7} \mathrm{~m}$ corresponds to the double layer. It looks like a single spike on this scale, but it is resolved in the simulation. The waves on the ion timescale seen in Fig. 4 are seen in Fig. $8 \mathrm{c}$ between $z=4.5 \times 10^{7} \mathrm{~m}$ and $z=5 \times 10^{7} \mathrm{~m}$, and they are also visible close to the double layer in Fig. 8a. In addition to these waves, there are also waves on the electron timescale. These appear near the local plasma frequency, and are seen in Fig. 8 both as wiggles on the electric field curve at high altitude and below the double layer at much shorter wavelength. Closeups of the lowaltitude, high-potential, side of the double layer are seen in Fig. $8 \mathrm{~b}$ and $\mathrm{d}$ for $t=1 \mathrm{~s}$ and $t=5 \mathrm{~s}$ respectively. We see that these waves are non-uniformly distributed in space, and that at $t=5 \mathrm{~s}$ they have been damped away in most of the region. In a small part of the region, near $z=5.46 \times 10^{7} \mathrm{~m}$, they remain with approximately the same amplitude they had at $t=1 \mathrm{~s}$. This can be understood by comparing the distribution functions of the magnetospheric electrons, which are shown in Fig. 9 for $z=5.10 \times 10^{7} \mathrm{~m}$ (solid line) and $z=5.46 \times 10^{7} \mathrm{~m}$ (dashed line). At $z=5.46 \times 10^{7} \mathrm{~m}$, where the waves remain undiminished, we have a bump on tail distribution, where the bump is formed by the precipitating electrons. At $z=5.10 \times 10^{7} \mathrm{~m}$ the bump is overshadowed by the trapped electron population. In Fig. 5a it is seen that the trapped distribution gets narrower in velocity as one goes from $z=5.10 \times 10^{7} \mathrm{~m}-$ that is to say, near the double layer - toward the ionosphere. 


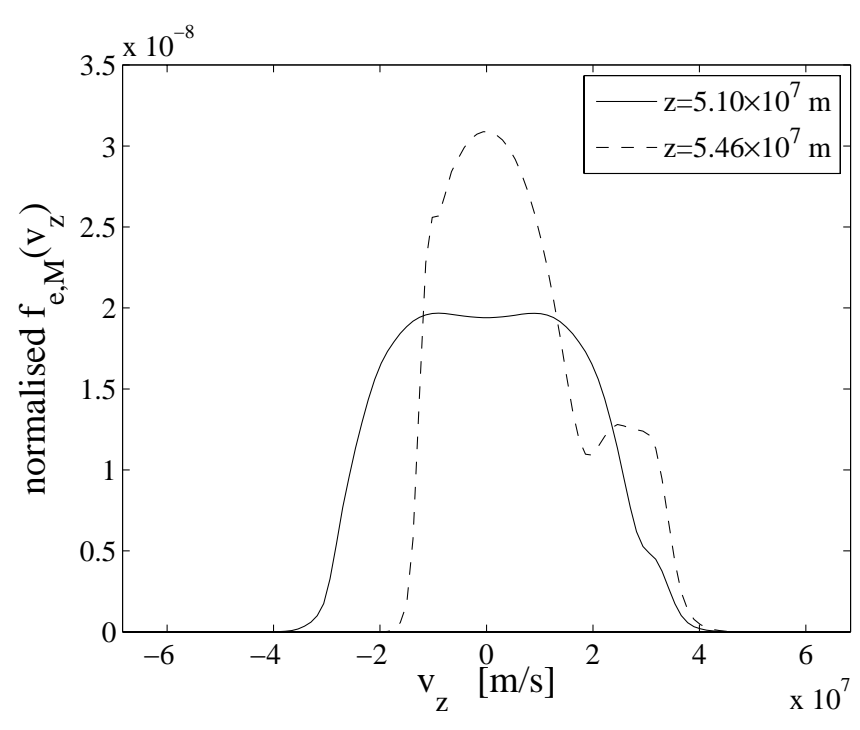

Fig. 9. Distribution function $f\left(v_{\mathrm{Z}}\right)$ of the electrons of magnetospheric origin for $z=5.10 \times 10^{7} \mathrm{~m}$ (solid line) and $z=5.46 \times 10^{7} \mathrm{~m}$ (dashed line). In both cases $t=5 \mathrm{~s}$. The distribution functions have been normalised so that $\int f\left(v_{\mathrm{Z}}\right) d v_{\mathrm{Z}}=1$.

\section{Conclusions and discussion}

We have modelled an auroral flux tube from the magnetospheric equator to the ionosphere by the use of Vlasov simulations. We have found that about two thirds of the total voltage is concentrated in a thin double layer, and that the remainder is located in an extended region above it. In this region waves arise due to an instability that is caused by the ionospheric ions that are accelerated in the double layer, forming a beam of ions that move away from the Earth. The double layer altitude is found to decrease with an increasing total voltage, and electrons of magnetospheric origin can become trapped between the double layer and the magnetic mirror.

An ion instability develops just above the double layer, as shown in Fig. 5d. Signs of the ion wave can be seen in the magnetospheric electron population, shown in Fig. 5a, at the same position as the perturbation of the ion beam. Waves near the local plasma frequency are also seen above the double layer, but these have no noticeable influence on the particle distributions. Wave activity from low frequencies up to the plasma frequency has been reported in the auroral cavity (Ergun et al., 2002), but we can not directly compare our calculations to the observations, since this would require a simulation model that includes more of the plasma wave modes.

Below the double layer, waves are present throughout its high-potential side. Close to the double layers these are transients in the beginning of the simulation, and they are soon damped away, whereas at lower altitude the waves can be maintained by a bump on tail instability. It develops as the trapped population is slowed down in $v_{\mathrm{z}}$ and re- flected by the magnetic mirror force. The precipitating electrons, which are more field aligned, and therefore less affected by the converging magnetic field, continue downward at high speed, forming the bump. The spatial distribution of these waves is non-uniform. Similar non-uniform distributions of waves have been observed before when electron beam-plasma interaction takes place in density gradients (Gunell and Löfgren, 1997; Löfgren and Gunell, 1998). Waves in this region are likely to contribute to the heating of the cold ionospheric electron population.

The double layer is located at lower altitudes for higher total voltages as is shown in Fig. 7b. This was also noticed by Boström (2004), who matched stationary quasi-neutral solutions with discontinuities, which account for the double layers. Boström (2004) also found that the position of the double layer depended on what assumptions were made about the presence of trapped electrons. This, together with the information that the formation of the double layer can trap electrons, suggests that there is an important interrelationship between trapped electron populations and the time-dependent potential profile.

Particles are trapped between the double layer and the magnetic mirror, and since we had no trapped population initially, one may ask how these particles became trapped. Equations (37) and (38) are derived assuming that $V(z)$ does not vary with time, and this assumption does not hold during the build-up of the potential structure. In a stationary state, trapped populations cannot reach the boundaries, and particles from the boundaries cannot be trapped. The steepening of the potential drop trapped the electrons during the buildup phase. In a stationary outstretched potential profile, electrons from the magnetosphere are accelerated by the electric field, and as they reach lower altitudes they are decelerated by the magnetic mirror force, which then accelerates them back upward. However, as the potential profile has changed and a larger fraction of the potential drop occurs at lower altitude, some of the electrons will not have gained enough energy from the mirror force before they are reflected downward again by the electric field, and thus they become trapped between the double layer and the magnetic mirror. In these simulations, the potential profile steepens as a result of the process of filling the system with plasma using a large $\epsilon_{\mathrm{r}}$, and then letting it decrease. In nature, any process which leads to potential steepening will be able to trap electrons, and since double layers do form in the auroral acceleration region, such processes must exist. Since the temporal evolution of the potential profile affects the ability to trap particles, the solution we arrive at is not unique. We have assumed that the flux tube is empty initially. Different initial conditions could lead to different equilibrium states. This was tested by comparing our standard way of initialising the system, i.e. letting it fill at $3 \mathrm{kV}$ and a high $\epsilon_{\mathrm{r}}$ value, which is then decreased in steps, to a different initialisation procedure where the system is filled with particles with $0 \mathrm{~V}$ applied and where the voltage subsequently is increased to $3 \mathrm{kV}$. Both ways of initialising 
the simulation yield nearly the same potential profile. The influence on the temporal evolution of the total voltage in the circuit and the density and temperature of the plasma sources at the magnetospheric and ionospheric ends of the system will be an interesting topic for future studies, since, in nature, these conditions do change with time.

According to Knight (1973) the current density is "independent of the behaviour of the electric potential as long as this does not have a significant minimum." The vast majority of the current is carried by electrons from the magnetosphere that populate region I in Fig. 6. It is seen in the figure that the boundary of region I, given by Eq. (37), follows the dashed curve closely, except in a scarcely populated region of panel (b). Panel (b) shows the distribution function at $z=4.91 \times 10^{7} \mathrm{~m}$, near which point there is a small local minimum, but since the affected area of phase space is unpopulated this does not influence the current. We see from the right-hand side of Eq. (37) that the factor $1 /\left(B(z)-B_{1}\right)$ has its minimum at the ionospheric end of the system, and since it spans several orders of magnitude, it will dominate Eq. (37) in most cases. For a minimum of the electric potential to influence the current it must not only reach a low enough value but also occur at low enough altitude. While being insignificant in determining the current, the shape of $V(z)$ is important for the ability to trap particles, as can be seen in Fig. $6 \mathrm{~b}$ and c, comparing the high- $\mu^{\prime}$ boundary of region III as computed according to Eq. (38) with the dashed lines that are based on $V$ and $B$ at the ends of the system.

The auroral current circuit can be considered as a system of four circuit elements: the upward current region, the ionosphere, the downward current region, and the generator. These different elements will have an influence on one another, in part because they are all part of the same circuit, and their respective properties regulate the current that flows in the circuit, but they also interact through means outside circuit theory. For example, the precipitating electrons contribute to heating of the ionosphere, and the properties of the ionosphere set the boundary conditions for the upward and downward current regions. The energy input into the circuit comes from the generator region, where plasma flows across the magnetic field will determine the voltage that is available to the aurora, and this can be modulated on different timescales (Haerendel, 2011).

In this work we have considered only one circuit element, namely the upward current region. This region is likely to carry a larger part of the voltage supplied by the generator than the opposite, downward, current region, as models predict the voltage across that region to be lower for the same current density (Vedin and Rönnmark, 2005). Observations of the downward current region also show comparatively low voltages of $1 \mathrm{kV}$ or less (Andersson et al., 2002, 2008). The same observations show that double layers in the downward current region often are unstable and that the field aligned potential drop can be variable rather than fixed. Thus, although the voltage in the downward current region is lower than in the upward current region, oscillations in the former can modulate the voltage that falls on the latter. This is likely to affect the potential profile and therefore the trapping of electrons in the upward current region. In the upward current region, the trapping and release of electrons can be expected to be of importance and a subject for future research.

\section{Supplementary material related to this article is available online at: http://www.ann-geophys.net/31/1227/ 2013/angeo-31-1227-2013-supplement.zip.}

Acknowledgements. This work was supported by the Belgian Science Policy Office through the Solar-Terrestrial Centre of Excellence and by PRODEX/Cluster PEA 90316. This research was conducted using the resources of the High Performance Computing Center North (HPC2N) at Umeå University in Sweden.

Topical Editor R. Nakamura thanks two anonymous referees for their help in evaluating this paper.

\section{References}

Alfvén, H. and Fälthammar, C.-G.: Cosmical Electrodynamics, Fundamental Principles, Clarendon Press, Oxford, second edn., 1963.

Andersson, L., Ergun, R. E., Newman, D. L., McFadden, J. P., Carlsson, C. W., and Su, Y.-J.: Formation of parallel electric fields in the downward current region of the aurora, Phys. Plasmas, 9, 3600-3609, doi:10.1063/1.1490134, 2002.

Andersson, L., Newman, D. L., Ergun, R. E., Goldman, M. V., Carlson, C. W., and McFadden, J. P.: Influence of suprathermal background electrons on strong auroral double layers: Observations, Phys. Plasmas, 15, 072901, doi:10.1063/1.2938751, 2008.

Boström, R.: Kinetic and space charge control of current flow and voltage drops along magnetic flux tubes: 2 . Space charge effects, J. Geophys. Res., 109, A01208, doi:10.1029/2003JA010078, 2004.

Brenning, N., Axnäs, I., Raadu, M. A., Tennfors, E., and Koepke, M.: Radiation from an electron beam in a magnetized plasma: Whistler mode wave packets, J. Geophys. Res., 111, A11212, doi:10.1029/2006JA011739, 2006.

Cheng, C. Z. and Knorr, G.: The integration of the Vlasov equation in configuration space, J. Comput. Phys., 22, 330-351, doi:10.1016/0021-9991(76)90053-X, 1976.

Chiu, Y. T. and Schulz, M.: Self-Consistent Particle and Parallel Electrostatic Field Distributions in the MagnetosphericIonospheric Auroral Region, J. Geophys. Res., 83, 629-642, doi:10.1029/JA083iA02p00629, 1978.

DeGroot, J. S., Barnes, C., Walstead, A. E., and Buneman, O.: Localized Structures and Anomalous dc Resistivity, Phys. Rev. Lett., 38, 1283-1286, doi:10.1103/PhysRevLett.38.1283, 1977.

De Keyser, J. and Echim, M.: Auroral and sub-auroral phenomena: an electrostatic picture, Ann. Geophys., 28, 633-650, doi:10.5194/angeo-28-633-2010, 2010. 
De Keyser, J., Maggiolo, R., and Echim, M.: Monopolar and bipolar auroral electric fields and their effects, Ann. Geophys., 28, 20272046, doi:10.5194/angeo-28-2027-2010, 2010.

Egedal, J., Daughton, W., Drake, J. F., Katz, N., and Lê, A.: Formation of a localized acceleration potential during magnetic reconnection with a guide field, Phys. Plasmas, 16, 050701, doi:10.1063/1.3130732, 2009.

Ergun, R. E., Carlsson, C. W., McFadden, J. P., Mozer, F. S., and Strangeway, R. J.: Parallel electric fields in discrete arcs, Geophys. Res. Lett., 27, 4053-4056, doi:10.1029/2000GL003819, 2000.

Ergun, R. E., Andersson, L., Main, D. S., Su, Y.-J., Carlsson, C. W., McFadden, J. P., and Mozer, F. S.: Parallel electric fields in the upward current region of the aurora: Indirect and direct observations, Phys. Plasmas, 9, 3685-3694, doi:10.1063/1.1499120, 2002.

Ergun, R. E., Andersson, L., Main, D. S., Su, Y.-J., Newman, D. L., Goldman, M. V., Carlsson, C. W., Hull, A. J., McFadden, J. P., and Mozer, F. S.: Auroral particle acceleration by strong double layers: The upward current region, J. Geophys. Res., 109, A12220, doi:10.1029/2004JA010545, 2004.

Filbet, F., Sonnendrücker, E., and Bertrand, P.: Conservative Numerical Schemes for the Vlasov Equation, J. Comput. Phys., 172, 166-187, doi:10.1006/jcph.2001.6818, 2001.

Fridman, M. and Lemaire, J.: Relationship between auroral electrons fluxes and field aligned electric potential difference, J. Geophys. Res., 85, 664-670, doi:10.1029/JA085iA02p00664, 1980.

Gunell, H. and Löfgren, T.: Electric Field Spikes Formed by Electron Beam-Plasma Interaction in Plasma Density Gradients, Phys. Plasmas, 4, 2805-2812, doi:10.1063/1.872413, 1997.

Gunell, H., Brenning, N., and Torvén, S.: Bursts of high-frequency plasma waves at an electric double layer, J. Physics D: Applied Physics, 29, 643-654, doi:10.1088/0022-3727/29/3/025, 1996a.

Gunell, H., Verboncoeur, J. P., Brenning, N., and Torvén, S.: Formation of Electric Field Spikes in Electron BeamPlasma Interaction, Phys. Rev. Lett., 77, 5059-5062, doi:10.1103/PhysRevLett.77.5059, 1996b.

Gurnett, D. A., Averkamp, T. F., Schippers, P., Persoon, A. M., Hospodarsky, G. B., Leisner, J. S., Kurth, W. S., Jones, G. H., Coates, A. J., Crary, F. J., and Dougherty, M. K.: Auroral hiss, electron beams and standing Alfvén wave currents near Saturn's moon Enceladus, Geophys. Res. Lett., 380, L06102, doi:10.1029/2011GL046854, 2011.

Haerendel, G.: Six auroral generators: A review, J. Geophys. Res. (Space Physics), 116, A00K05, doi:10.1029/2010JA016425, 2011.

Hull, A. J., Bonnell, J. W., Mozer, F. S., Scudder, J. D., and Chaston, C. C.: Large parallel electric fields in the upward current region of the aurora: Evidence for ambipolar effects, J. Geophys. Res., 108, 1265, doi:10.1029/2002JA009682, 2003.

Hwang, K.-J., Ergun, R. E., Newman, D. L., Tao, J.-B., and Andersson, L.: Self-consistent evolution of auroral downward-current region ion outflow and a moving double layer, Geophys. Res. Lett., 36, L21104, doi:10.1029/2009GL040585, 2009.

Ishiguro, S., Kishi, Y., and Sato, N.: Potential Formation due to Plasma Injection Along Converging Magnetic Field Lines, Phys. Plasmas, 2, 3271-3274, doi:10.1063/1.871161, 1995.

Knight, S.: Parallel Electric Fields, Planet. Space Sci., 21, 741-750, doi:10.1016/0032-0633(73)90093-7, 1973.
Löfgren, T. and Gunell, H.: Flexible Simple-Pole Expansion of Distribution Functions, Phys. Plasmas, 4, 3469-3476, doi:10.1063/1.872243, 1997.

Löfgren, T. and Gunell, H.: Interacting eigenmodes of a plasma diode with a density gradient, Phys. Plasmas, 5, 590-600, doi:10.1063/1.872751, 1998.

Lyons, L. R.: Generation of large-scale regions of auroral currents, electric potentials, and precipitation by the divergence of the convection electric field, J. Geophys. Res., 85, 17-24, doi:10.1029/JA085iA01p00017, 1980.

Main, D. S., Newman, D. L., and Ergun, R. E.: Double Layers and Ion Phase-Space Holes in the Auroral Upward-Current Region, Phys. Rev. Lett., 97, 185001, doi:10.1103/PhysRevLett.97.185001, 2006.

Marklund, G. T., Sadeghi, S., Karlsson, T., Lindqvist, P.-A., Nilsson, H., Forsyth, C., Fazakerley, A., Lucek, E. A., and Pickett, J.: Altitude Distribution of the Auroral Acceleration Potential Determined from Cluster Satellite Data at Different Heights, Phys. Rev. Lett., 106, 055002, doi:10.1103/PhysRevLett.106.055002, 2011.

Newman, D. L., Andersson, L., Goldman, M. V., Ergun, R. E., and Sen, N.: Influence of suprathermal background electrons on strong auroral double layers: Vlasov-simulation parameter study, Phys. Plasmas, 15, 072902, doi:10.1063/1.2938753, 2008a.

Newman, D. L., Andersson, L., Goldman, M. V., Ergun, R. E., and Sen, N.: Influence of suprathermal background electrons on strong auroral double layers: Laminar and turbulent regimes, Phys. Plasmas, 15, 072903, doi:10.1063/1.2938754, 2008b.

Persson, H.: Electric Field Parallel to the Magnetic Field in a Low-Density Plasma, Phys. Fluids, 9, 1090-1098, doi:10.1063/1.1761807, 1966.

Pierrard, V.: New model of magnetospheric currentvoltage relationship, J. Geophys. Res., 101, 2669-2675, doi:10.1029/95JA00476, 1996.

Pierrard, V., Khazanov, G., and Lemaire, J.: Current-voltage relationship, J. Atmos. Solar-Terr. Phys., 69, 2048-2057, doi:10.1016/j.jastp.2007.08.005, 2007.

Rönnmark, K. and Hamrin, M.: Auroral electron acceleration by Alfvén waves and electrostatic fields, J. Geophys. Res., 105, 25333-25344, doi:10.1029/2000JA900103, 2000.

Sato, N., Nakamura, M., and Hatakeyama, R.: Three-Dimensional Double Layers Inducing Ion-Cyclotron Oscillations in a Collisionless Plasma, Phys. Rev. Lett., 57, 1227-1230, doi:10.1103/PhysRevLett.57.1227, 1986.

Sato, N., Watanabe, Y., Hatakeyama, R., and Mieno, T.: Potential Formation in a High-Speed Plasma Flow along Converging Magnetic Field Lines, Phys. Rev. Lett., 61, 1615-1618, doi:10.1103/PhysRevLett.61.1615, 1988.

Sato, T. and Okuda, H.: Ion-Acoustic Double Layers, Phys. Rev. Lett., 44, 740-743, doi:10.1103/PhysRevLett.44.740, 1980.

Sato, T. and Okuda, H.: Numerical Simulations on Ion Acoustic Double Layers, J. Geophys. Res., 86, 3357-3368, doi:10.1029/JA086iA05p03357, 1981.

Sato, T., Takamaru, H., and The Complexity Simulation Group: Kinetic self-organization: Creation of super ion acoustic double layer, Phys. Plasmas, 2, 3609-3613, doi:10.1063/1.871442, 1995.

Schmitz, H. and Grauer, R.: Darwin Vlasov simulations of magnetised plasmas, J. Comput. Phys., 214, 738-756, 
doi:10.1016/j.jcp.2005.10.013, 2006.

Schrittwieser, R., Axnäs, I., Carpenter, T., and Torvén, S.: Observation of double layers in a convergent magnetic field, IEEE Transactions on Plasma Science, 20, 607-613, doi:10.1109/27.199500, 1992.

Song, B., Merlino, R. L., and D'Angelo, N.: On the stability of strong double layers, Physica Scripta, 45, 391-394, doi:10.1088/0031-8949/45/4/018, 1992a.

Song, B., Merlino, R. L., and D'Angelo, N.: The effect of a magnetic field gradient on anode double layers, Physica Scripta, 45, 395-398, doi:10.1088/0031-8949/45/4/019, 1992 b.

Torvén, S.: High-voltage double layers in a magnetised plasma column, Journal of Physics D: Applied Physics, 15, 1943-1949, doi:10.1088/0022-3727/15/10/012, 1982.

Torvén, S. and Andersson, D.: Observations of electric double layers in a magnetised plasma column, J. Physics D: Applied Physics, 12, 717-722, doi:10.1088/0022-3727/12/5/012, 1979.

Vedin, J. and Rönnmark, K.: A linear auroral current-voltage relation in fluid theory, Ann. Geophys., 22, 1719-1728, doi:10.5194/angeo-22-1719-2004, 2004.
Vedin, J. and Rönnmark, K.: Electrostatic potentials in the downward auroral current region, J. Geophys. Res., 110, 8207, doi:10.1029/2005JA011083, 2005.

Vedin, J. and Rönnmark, K.: Particle-fluid simulation of the auroral current circuit, J. Geophys. Res., 111, 12201, doi:10.1029/2006JA011826, 2006.

Watt, C. E. J. and Rankin, R.: Electron Trapping in Shear Alfvén Waves that Power the Aurora, Phys. Rev. Lett., 102, 045002, doi:10.1103/PhysRevLett.102.045002, 2009.

Watt, C. E. J. and Rankin, R.: Do magnetospheric shear Alfvén waves generate sufficient electron energy flux to power the aurora?, J. Geophys. Res., 115, A07224, doi:10.1029/2009JA015185, 2010.

Watt, C. E. J., Rankin, R., and Marchand, R.: Kinetic simulations of electron response to shear Alfvén waves in magnetospheric plasmas, Phys. Plasmas, 11, 1277-1284, doi:10.1063/1.1647140, 2004.

Whipple Jr., E. C.: The Signature of Parallel Electric Fields in a Collisionless Plasma, J. Geophys. Res., 82, 1525-1531, doi:10.1029/JA082i010p01525, 1977. 